\title{
Intermédialités
}

Histoire et théorie des arts, des lettres et des techniques

Intermediality

History and Theory of the Arts, Literature and Technologies

\section{Le portrait en malade. Histoire de sa face cachée}

\section{Florence Chantoury-Lacombe}

Numéro 8, automne 2006

Envisager

Facing

URI : https://id.erudit.org/iderudit/1005538ar

DOI : https://doi.org/10.7202/1005538ar

Aller au sommaire du numéro

Éditeur(s)

Centre de recherche sur l'intermédialité

ISSN

1705-8546 (imprimé)

1920-3136 (numérique)

Découvrir la revue

Citer cet article

Chantoury-Lacombe, F. (2006). Le portrait en malade. Histoire de sa face cachée. Intermédialités / Intermediality, (8), 31-51.

https://doi.org/10.7202/1005538ar
Résumé de l'article

Cette étude consacrée à l'interprétation du portrait en malade tente de démontrer comment, à travers la littérature artistique de la Renaissance et dans le discours des historiens de l'art contemporains, le portrait représentant un personnage malade a fait l'objet d'une euphémisation dans laquelle une bonne santé de la peinture était privilégiée au détriment d'une interprétation plus probante du portrait pathologique. Par l'analyse de différents portraits de gens varioles, syphilitiques ou atteints d'autres pathologies à la Renaissance, il s'agit d'observer que la représentation du visage pathologique met à mal le statut même du portrait. 


\title{
Le portrait en malade Histoire de sa face cachée
}

\author{
Florence Chantoury-lacombe
}

«Il n’est pas exclu que la lèpre soit désormais

nécessaire pour apprécier la Joconde ${ }^{1}$.»

e portrait en malade a souvent été interprété comme un objet qui contrevient à l'idée convenue de ce que doit être l'art; il retire à l'image son apparente évidence et la rend soudainement problématique. Le portrait d'une pathologie suscite éventuellement une indignation théorique et renvoie à l'interdit de la figuration, à l'angoisse du contrôle de soi. Le portrait a été soumis à des usages politiques, il est une mise en évidence théâtrale d'un individu dans laquelle l'artiste se rallie en partie à une formule qui satisfait le commanditaire. Mais le portrait déborde largement la simple mise en évidence de son modèle, il offre une définition de la personne représentée par le décor, la pose, le matériau utilisé et signale l'appartenance à un groupe ou à une classe. Nous pouvons imaginer que le modèle, souvent commanditaire de l'œuvre, aspirait à une pose qui l'avantageait et qui respectait les lois du genre telles qu'elles se formulaient à la Renaissance. Il voulait voir dans son portrait les normes en vigueur qui pouvaient dépendre des hiérarchies en vogue, des variations d'une psychologie, d'une caractériologie sensible à ce qui apparente une personne aux membres de son groupe, ou au contraire à ce qui l'en distingue.

Nous devons donc déjà supposer qu'un régime de l'idéalisation traverse le portrait de la Renaissance. Nous en avons un cas significatif avec les divers portraits de Federico da Montefeltre, que nous a légués la peinture du quattrocento

1. Jean-Marie Pontévia, Tout a peut-être commencé par la beauté, Préface de Gérard Grand, Bordeaux, William Blake and Co., 2001, p. 61. 
témoignant de cette volonté de dissimulation du fait pathologique². La représentation récurrente du profil de Montefeltre correspond à une volonté d'idéalisation, relevant dans ce cas de la détermination à ne pas montrer les défauts du Duc d'Urbino en dissimulant la marque de la perte de l'œil droit33. Les quatre portraits les plus connus de Federico da Montefeltre répondent aux principes d’idéalisation énoncés par Alberti. Les portraits de Montefeltre exposent de ce que l'on pourrait nommer une histoire secrète de la peinture dérobante. Le duc de Montefeltre avait perdu son œil droit dans une joute et le fait que le duc était borgne n'est jamais dévoilé dans ses portraits, l'accident de l'œil n'est pas montré, mais suggéré par la représentation picturale du nez, tranché par un coup d'épée dans ce même accident. Les portraits connus du duc - et notamment celui de Piero della Francesca (fig. 1) - utilisent tous les conventions antiques de la peinture de portraits par l'application pratique du topos d'Antigone Le Borgne, à savoir peindre le modèle de profil, en vue d'offrir une solution figurative au

2. Nous rappelons ici l'un des passages d'Alberti: «Que les parties honteuses du corps et toutes les parties peu gracieuses soient couvertes d'un linge, d'un feuillage ou de la main. Apelle ne peignit l'image d'Antigone que du côté du visage où le défaut de l'œil n'apparaissait pas. On dit que Périclès avait une tête allongée et difforme; c'est pourquoi les peintres et les sculpteurs ne le montraient pas tête nue, comme les autres, mais coiffé d'un casque. Plutarque rapporte aussi que les peintres anciens avaient l'habitude, lorsqu'ils peignaient des rois, s'ils avaient quelque défaut de ne pas donner l'impression d'avoir voulu l'omettre mais, autant qu'ils le pouvaient, ils le corrigeaient tout en maintenant la ressemblance. Je désire donc que cette modestie et cette retenue soient observées dans toute histoire afin que ce qui est choquant soit omis ou corrigé. » (Leon Battista Alberti, De Pictura, De pictura/De la peinture, Traduction et notes de Jean-Louis Schefer, Paris, Macula/Dédale, 1992, p. 173) On retrouve également cette requête chez Plutarque, dans la vie d'Agésilas, $\mathbb{2}$ 2-4, «Nous n'avons aucun portrait de lui (il n'en voulait pas et défendait même en mourant que son aspect physique fut représenté par la sculpture ou par tout autre procédé), mais l'on dit qu'il était petit et de physionomie médiocre. » (Plutarque, Vies, tome 8, texte établi et traduit par Robert Flacelière et Emile Chambry, Paris, Éditions Les Belles Lettres, 1973, p. 97) Également chez Pline l’Ancien, Histoire naturelle, livre XXXV, texte établi, traduit et commenté par Jean-Michel Croisille, Paris, Les Belles Lettres, 1985, \&90, p. 75. Voir aussi Joanna Woods-Marsden, "Ritratto al Naturale": Questions of Realism and Idealism in Early Renaissance Portraits ", dans Art Journal, vol. $46, n^{\circ} 3,1987$, p. 209-216. Le désir d'annulation de toute difformité se retrouve également chez Dolce, Armenini et Biondo.

3. Cet événement est rapporté par Martin Warnke dans Lartiste et la cour: aux origines de l'artiste moderne, Paris, Éditions de la Maison des Sciences de l'Homme, 1995, p. 264. Il apparaît également dans la biographie que Vespasiano da Bisticci consacre au Duc de Montefeltre. Voir «Vita di Federico da Montefeltre », par Vespasiano da Bisticci, dans Vite di Uomini Illustri del secolo XV, Florence, $193^{8}$. 


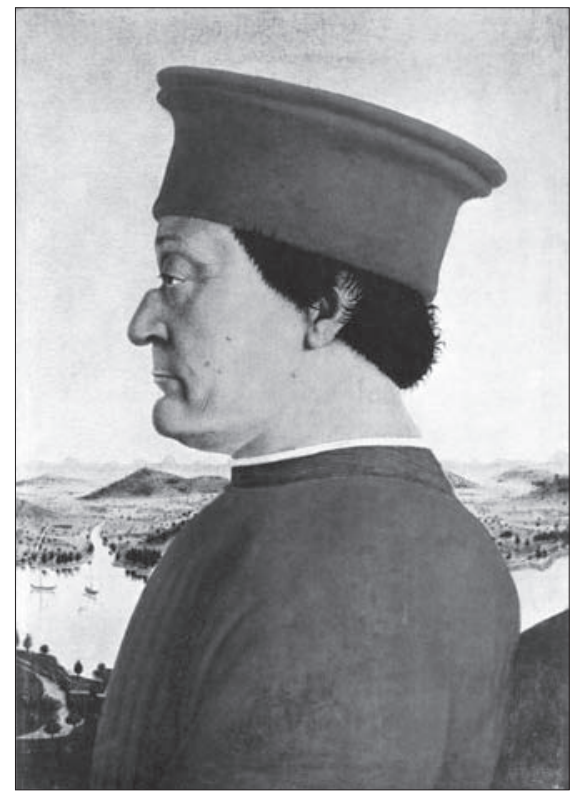

Fig. 1. Piero della Francesca, Frederico da Montefeltre, 1466, détrempe sur panneau, Florence, Galerie des Offices. (C) Avec l'aimable autorisation du Ministero dei Beni e le Attività Culturali.

précepte d'Alberti concernant l'adoucissement du défaut. De la même manière, la malformation faciale de Charles Quint, le menton proéminent qui entravait la fermeture de la bouche et dont plusieurs contemporains ont décrit l'aspect, n'apparaît pas non plus dans les portraits de l'empereur' ${ }^{4}$. Le portraitiste, lorsqu'il

4. Voir la description de l'ambassadeur vénitien Gaspare Contarini en 1525 résumée dans les Diarii de Marino Sanudo, XL, col. 289. Le diagnostic posé par la médecine moderne est celle d'une prognathie mandibulaire. Pour une étude du prognathisme de Charles Quint d'un point de vue médical, voir Oswald Rubbrecht, L'origine du type familial de la maison de Habsbourg, Bruxelles, G. Van Oest, 1910. Également, Félix Regnault, «Charles-Quint devant la médecine» dans Le correspondant médical, 15 août 1995, p. 5. L'iconodiagnostic utilisé par Diane Bodart dans son étude des portraits de Charles Quint permet, à travers le prognathisme, d'aborder la question de la ressemblance dans ces portraits. L'historienne de l'art endosse ici la blouse du médecin pour poser un diagnostic rétrospectif. Selon cet auteur, l'exaltation des stigmates familiaux affirme la légitimité de la continuité dynastique. Diane Bodart, Pouvoirs du portrait sous l'empire des Habsbourg d'Espagne. 1500-1700, Thèse sous la direction de Daniel Arasse, EHESS, 2003, voir chapitre 3, p. 111 et 105. 


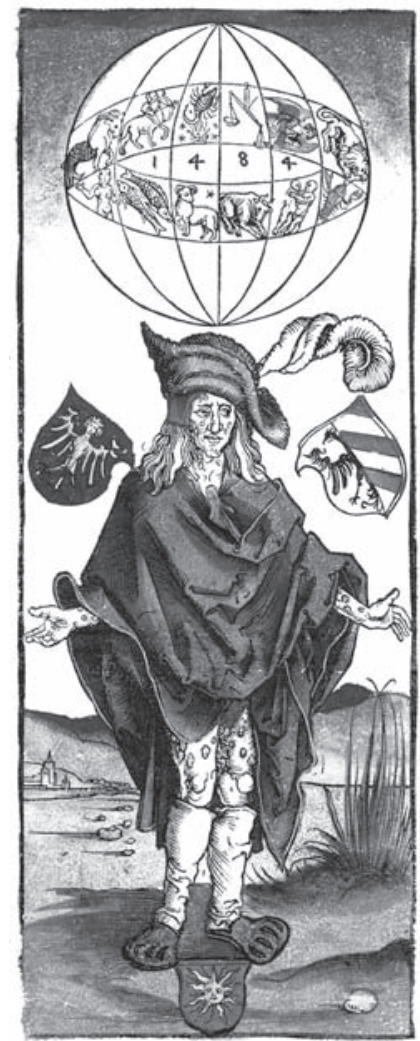

Fig. 2. Albrecht Dürer, L'homme syphilitique, 1496, gravure sur bois, illustration de l'ouvrage de Theodoricus Ulsenius, Vaticinium in epidemicam scabiem, Nuremberg. Kupferstichkabinett, Staatliche Museen zu Berlin, Berlin, Allemagne. (C) Bildarchiv Preussischer Kulturbesitz / Art Resource, New York.

dissimule la maladie, ne fait pas un aveu d'indifférence, il exclut l'élément perturbateur qui court-circuiterait la fonction du portrait5. Car le portrait tire avantage de la curiosité que provoque toute figure humaine, sa portée esthétique vient par surcroît, elle se déploie là où le modèle devient, pour le peintre, l'annonce d'une forme. La théorie du portrait se devrait donc d'avoir sa part maudite, elle devrait aussi avoir une histoire de sa face cachée, de la face cachée du visage. Mais il ne

5. En reprenant l'enseignement des traités physiognomoniques de l'Antiquité, les auteurs de la Renaissance ont aussi souligné que la difformité du visage est toujours reconnue comme le signe d'une mauvaise nature. Le personnage de Judas dans La Cène de Léonard fait souvent office d'exemple. 
faut pas tomber dans l'écueil de voir dans la peinture un mandat d'obligation de manifester les caractères d'un personnage et donc de vouloir à tout prix observer la pathologie qui traverse son corps; c'est demander ici une exigence de peindre la maladie. Faire dire à la peinture qu'elle est dans l'obligation de tout représenter, c'est faire d'elle un lieu d'authenticité dont la fonction serait encore une fois la ressemblance. Pourtant, il existe bien un tabou dans la représentation d'un type de pathologie. Nous prendrons pleinement la dimension véritable de cette face cachée par la peinture mais aussi de la peinture lorsque nous confronterons la production picturale de la Renaissance à la nouvelle maladie dévastatrice qui fait son entrée sur la scène européenne à la fin du Xve siècle : la syphilis.

Au mois de décembre de l'année 1494 il se développa en Italie presque toute entière une maladie de nature jusqu'alors inconnue. Cette maladie reçut des divers peuples qu'elle affligea des dénominations différentes. Elle fut appelée mal de Naples par les Français, qui prétendirent l'avoir contractée à Naples et l'avoir rapportée de là dans leur pays. Les Napolitains, de leur côté, lui donnèrent le nom de mal français, parce qu'elle s'était manifestée et répandue pour la première fois en Italie à l'époque de l'expédition française. Les Génois l'appelèrent encore lo male de la tavelle, les Toscans lo male de le bulle, les Lombards, lo male de le brosule, les Espagnols las buas ${ }^{6}$.

Ce passage du médecin de Jules II, Giovanni da Vigo, souligne le caractère épidémique de cette maladie auparavant inexistante, la syphilis. Il indique le glissement nominatif dont elle a été l'enjeu car elle portait le nom du voisin détesté, ce qui renvoie à la question de l'identité nationale d'une maladie?. Son nom actuel de syphilis provient d'un poème de Girolamo Fracastoro, archiatre de Paul III et médecin du Concile de Trente, Syphilis sive de morbo Gallico, paru en 1530 à Vérone et dédicacé à Pietro Bembo. La syphilis a été définie par Fracastoro

6. Giovanni da Vigo, médecin de Jules II, fait paraître en 1514 une Pratica in arte chirurgica. Extrait du livre V.

7. La liste peut être encore étendue si l'on considère que, pour les Polonais, il s'agissait du mal allemand et, pour les Russes, du mal polonais. Lorigine de la syphilis pose encore de nombreux problèmes au monde de la médecine. Ce qui fut considéré comme une épidémie de syphilis en 1493 laisse encore en suspens la question de savoir s'il s'agissait bien de la syphilis. La virulence et la sévérité des symptômes ne correspondent pas avec la nosologie actuelle de la syphilis. Certains historiens de la médecine pensent que Christophe Colomb a peut-être rapporté une autre maladie de contamination vénérienne qui aurait disparu après quelques décennies. À ce sujet, voir Olivier Dutour, György Palfi, Jacques Bérato et Jean-Pierre Brun (dirs.), Lorigine de la syphilis en Europe: avant ou après 1493?, Actes du colloque international de Toulon, 25-28 novembre 1993, Toulon, Centre archéologique du Var, Paris, Errance, 1994. 
à travers son aspect contagieux qui se propage par le coït et qui se caractérise au début par des petites ulcérations aux parties génitales, suivies d'éruption de pustules le plus souvent croûteuses au cuir chevelu et par tout le corps. Fracastoro relève les importants dégâts causés dans la bouche, sur le voile du palais et dans le pharynx. Le poème de Fracastoro est un condensé de l'ensemble des thérapeutiques usitées à l'époque et les explications théologiques et astrologiques de l'origine du mal ne sont pas évacuées du livre. Erwin Panofsky a consacré un article à la représentation de la syphilis dans lequel il forge une iconographie de la syphilis tout entière dépendante de l'ouvrage de Fracastoro ${ }^{8}$. L'article s'organise autour de deux grandes parties : la première est consacrée à l'origine des descriptions littéraires de la syphilis, dans laquelle l'ouvrage de Girolamo Fracastoro acquiert une place prépondérante. À travers l'ouvrage de Fracastoro, Panofsky étudie la vision qui est donnée de cette maladie, les causes et la description des symptômes. Il relate le caractère de punition qu'elle revêt, la prophylaxie mise en place à la Renaissance et le traitement au bois de gaïac. Panofsky souligne le caractère comique que prend le savoir commun autour de la syphilis dans la seconde moitié du XVI ${ }^{\mathrm{e}}$ siècle et son essor au XVIII ${ }^{\mathrm{e}}$ siècle. La seconde partie du texte est réservée à l'étude de la représentation de la syphilis. Panofsky a la conviction d'observer le même schéma progressiste dans la figuration de la maladie. Ainsi, dans la production picturale de la Renaissance, le premier stade d'intérêt des artistes pour la syphilis est d'ordre théologique et astrologique pour s'achever avec la série d'Hogarth, Le mariage à la mode de 1745. Selon Panofsky, c'est Dürer qui inaugure ce moment par une gravure sur bois, L'homme syphilitique de 1496 (fig. 2). D'après l'historien de l'art allemand, Dürer donne une explication astrologique de la syphilis. Panofsky décrit la partie supérieure de l'image, occupée par un large globe céleste sur lequel nous retrouvons les signes du zodiaque et l'inscription de l'année 1484 . Tout cela indique la conjonction cette année-là de la concentration de cinq planètes dans le signe du scorpion, signes interprétés comme annonciateurs de la syphilis. Panofsky observe les armes de la ville de Nuremberg et le geste du personnage qu'il interprète comme une parodie ludique de l'ostentatio vulnerum. D’après Erwin Panofsky, le costume, jugé extravagant, caractérise certainement un «Frenchman». Lorsque Panofsky aborde le dessin d'Holbein (fig. 3), il souligne d'emblée le caractère unique de ce portrait qui allie, selon lui, une impitoyable objectivité et une compréhension compatissante devant le visage d'un jeune homme syphilitique. Panofsky observe que la pathologie a été identifiée à diverses maladies, telles que la lèpre ou encore à une éruption cutanée de la peau appelée impetigo contagiosa, mais le diagnostic

8. Erwin Panofsky, «Homage to Fracastoro in a Germano-Flemish Composition of about 1590? ", Nederlands Kunsthistorisch Jaarboek, n ${ }^{\circ}$ 12, 1961, p. 1-33. 


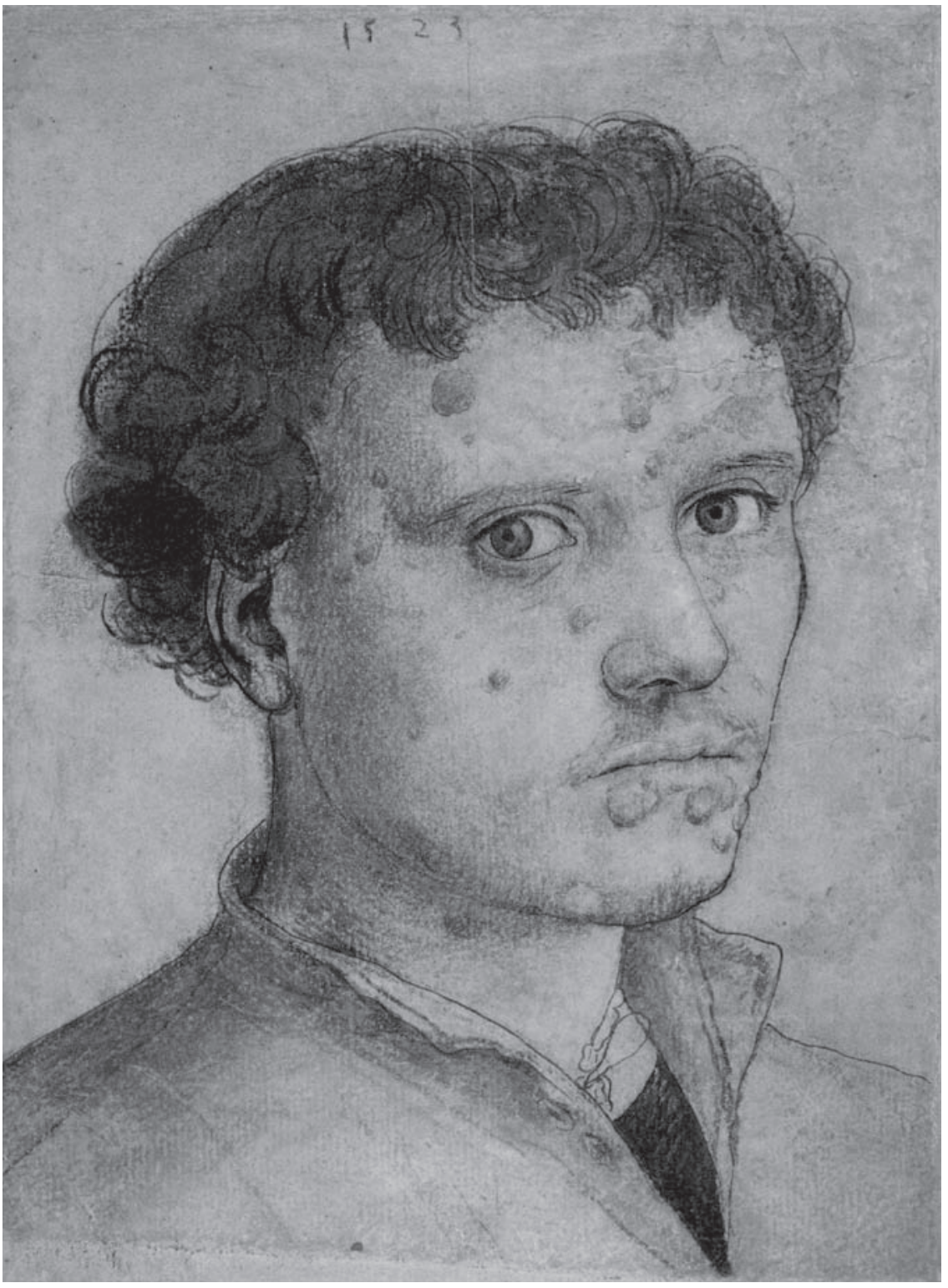

Fig. 3. Holbein le Jeune, Portrait d'Ulrich von Hutten, 1523, dessin, 20,5 x 15,2 cm. Cambridge, Fogg Art Museum. (C) Avec l'aimable autorisation du Fogg Art Museum, Harvard University Museums, Bequest of Paul J. Sachs, «A Testimonial to my Friend Felix M. Warburg». 
exact apparaît, selon lui, lorsque nous confrontons le dessin d'Holbein à l'image de Dürer, car il présente les mêmes symptômes. Tout cela se trouve confirmé par une inscription disparue sur le dessin même de Holbein : «Porträt U. von Hutten in seinem Todesjahr». Au recto du portrait, la date de 1523 correspond, selon Panofsky, à la date du décès de von Hutten. Le chevalier Ulrich de Hutten, diplomate et théologien allemand, aurait contracté la syphilis lorsqu'il servait en Italie comme lansquenet. Dans son ouvrage De Gaïaci Medicina et Morbo Gallico, publié à Mayence en 1519, il raconte l'histoire de sa guérison et présente le bois de gaïac comme le nouveau traitement plus efficace que les cures mercurielles 9 . Le long témoignage écrit des traitements successifs de la syphilis endurés par Von Hutten permet à Panofsky de faire de la maladie un alibi biographique.

L'histoire de la représentation de la syphilis se règle par divers moments selon Erwin Panofsky. Les images liminaires qui dépeignent la syphilis se caractérisent par une sobre description de la maladie, souvent bienveillante, puis vient ensuite une caractérisation des faits cliniques. Après que la nature vénérienne de la syphilis ait été signifiée par Dürer et Holbein, les peintres procèdent à des allégories et laissent place à la satire sociale, dans la dernière phase des peintures de syphilis étudiées par l'historien de l'art de Princeton. Au moyen de sa lecture iconographique, Erwin Panofsky contourne la question d'une pénurie d'images de la syphilis en identifiant divers types de représentations, astrologiques, satiriques, moralisantes ou biographiques. Par un postulat référentiel demandé à l'image, Panofsky a vite fait de constater que cette manière d'étudier la représentation de la syphilis est caduque. Il recherche alors d'autres types d'images, qui vont trouver une légitimité par le fait qu'ils sont associés aux diverses conceptions de la maladie qu'avait Fracastoro.

Panofsky n’a pas voulu suggérer que la pathologie de la syphilis pose des limites à la peinture. Pourtant, avec la syphilis, nous touchons un problème théorique de la représentation de la maladie, car si les symptômes sont extrêmement diversifiés ils ne sont pas toujours repérables. La syphilis est un état pathologique à plusieurs phases et dont le caractère principal est d'être, justement, un processus. Toutefois, la rareté des images cliniques de syphilis ne s'explique pas par une peur envers la syphilis. S'il faut reconnaître très tôt un discours moral dévolu à la syphilis, il ne s'agit pas d'y voir les enjeux sociaux du XIx ${ }^{\mathrm{e}}$ siècle dans lequel la syphilis apparaît sous l'angle du ressentiment moral, telle une maladie honteuse et dont la bourgeoisie chrétienne a fait le repoussoir de la moralité.

9. Par analogie avec les traitements des maladies de peau, le mercure fut employé pour soigner la syphilis. Parfois le mercure était appliqué en cérats sur la jambe ou sur le bras. Voir Antoine Marmottans, «Les traitements anciens de la syphilis », dans Lorigine de la syphilis en Europe: avant ou après 1493?, p. 258. 
L'histoire de la syphilis en peinture est l'histoire d'un vide dans la représentation qui a laissé place au supplément, tel que l'entendait Jacques Derrida. La peinture du portrait va s'adapter à la syphilis en déguisant le corps au moyen de perruques, gants, fards et poudres qui sunissent pour cacher les marques syphilitiques. Ces accessoires de dissimulation vont permettre aux peintres de faire preuve de convenance dans les portraits.

\section{COMME LE NEZ AU MILIEU DE LA FIGURE}

Le portrait de malade incontestablement le plus connu dans le champ de l'histoire de l'art est l'œuvre de Domenico Ghirlandaio, Le vieil homme et l'enfant, daté de 1490. En fait, il s'agit d'un double portrait dans lequel le peintre a représenté, face à face, un vieil homme et un enfant (fig. 4). Le vieillard est atteint d'une maladie qualifiée par les médecins d'acné hypertrophique, localisé autour du nez. Il s’agit d'excroissances qui ont déformé la paroi nasale. Aussi nommé rhinophyma, il s'agit du stade ultime de l'acné rosacé qui touche plutôt les personnes âgées. Bernard Berenson évoque la scène représentée comme celle d'un vieil homme tenant un enfant à distance de bras afin de le regarder intensément avant de l'embrasser. Il ajoute qu'il n'existe pas dans toute la peinture du quattrocento, ni en Italie ni ailleurs, une représentation si élevée d'un sentiment aussi humain ${ }^{10}$. Bernard Berenson décrit le personnage du vieillard de la manière suivante :

Le front est large et il a un je ne sais quoi de rocheux. Le trait le plus remarquable est le nez qui se révèle dans le visage entier. Le nez est bosselé, froncé et rugueux tel qu'il ressemble à un coing ou à une pomme de terre dans sa première croissance. Toutefois, cette singulière difformité ne répugne pas et elle ne diminue pas la dignité du visage ${ }^{11}$.

Le détail du nez donné pour ressemblant cautionne l'effet de présence. La maladie, cet élément extérieur qui n’entre pas dans le schéma habituel du dispositif du portrait, trouve une place ici pour rehausser la candeur et la grâce de

10. "Non esiste in tutta la pittura del Quattrocento, nè in Italia nè all'estero, un altro quadro pieno al pari di questo di un sentimento così schiettamente umano.» (Bernard Berenson, «Nova Ghirlandajana », L'arte, vol. 36, 1933, p. 175)

11. «La fronte è larga ed ha un che di roccioso. Il trattamento più saliente è il naso chi se rivele nel intera maschera. Il naso è bitorzoluto increspato e rugoso che somiglia ad un mela cotogna o a una patata nella prima crescenza. Però non è repellente questa singolare deformità e non menoma la dignità del volto.» (Bernard Berenson, «Nova Ghirlandajana », p. 175) 


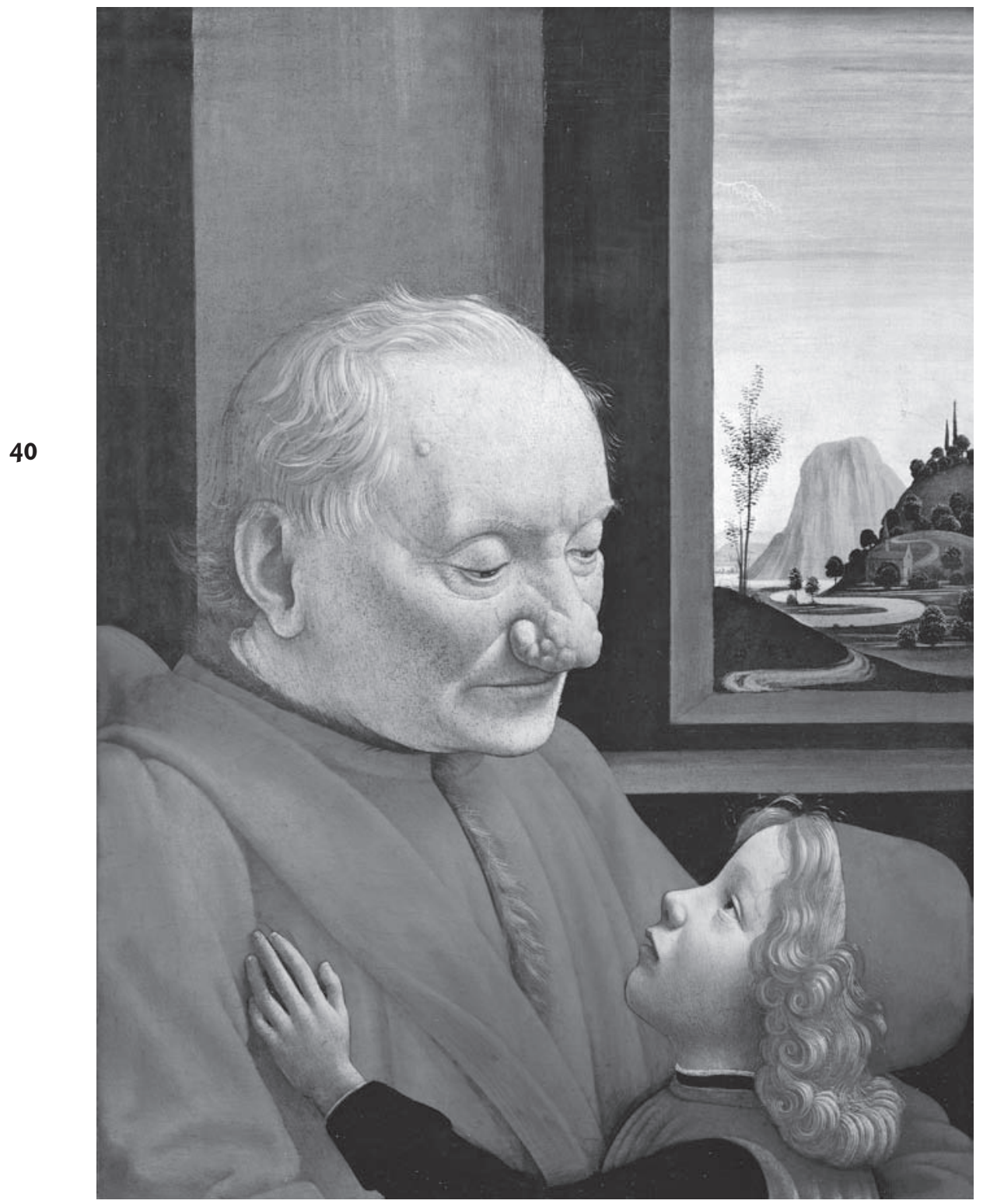

Fig. 4. Domenico Ghirlandaio, Le vieil homme et l'enfant, 1490, détrempe sur bois, 62,7 x 46,3 cm, Paris, Louvre. (c) Musée du Louvre / Art Resource, New York. 
l'enfant, ainsi le tableau révèle le «génie » de Ghirlandaio qui consiste à prêter une attention soutenue à une pathologie et à une difformité du visage, en évitant la caricature qui accuse jusqu'au ridicule des traits parfois peu flatteurs. D’après plusieurs historiens de l'art qui se sont penchés sur le portrait du vieillard, le peintre parvient à donner un statut à la peinture en utilisant la pathologie du malade et le système représentatif de la Renaissance, c'est-à-dire en produisant une mise en scène du portrait. Selon eux, l'artiste choisit d'obéir aux normes du portrait en vigueur en refusant un seul interdit, celui de la figuration de la maladie. Ruse ou génie de l'artiste, Ghirlandaio accepte le système représentatif, mais il le pratique avec une si grande maîtrise que le portrait pathologique est hissé au-dessus des œuvres ordinaires et donne un prestige à la déformation. En étudiant les mécanismes de la représentation, les historiens de l'art tentent de montrer de quelle manière l'artiste a produit un portrait singulier, imprévu, choquant mais néanmoins acceptable. L'ordre d'évidence par lequel l'histoire de l'art envisage le plus souvent le genre du portrait se trouve mis à mal avec le tableau de Ghirlandaio.

\section{L'INVENTION D'UNE SCÉNOGRAPHIE}

Un dessin à la pointe d'argent est considéré, par la plupart des auteurs, comme préparatoire au tableau. Les avis des spécialistes divergent sur la pose réelle du vieillard, plutôt sur l'état physique du corps, mort ou endormi. Berenson le juge comme l'un des meilleurs dans la production de l'artiste, bien qu'il reste inférieur par rapport au tableau. Il voit l'étude d'un homme endormi dans le dessin, comme Lorne Campbell, dans Renaissance Portraits, croit à l'hypothèse du vieillard endormi ${ }^{12}$. L'interprétation du dessin par John Pope-Hennessy tente de mettre en lumière les éléments qui ont permis au peintre de faire passer le modèle mort pour un vivant. Il souligne que le dessin révèle que le sujet n'a pas été dessiné de son vivant mais à partir du corps mort déposé dans la bière ${ }^{13}$. La tête dans le dessin préliminaire a été adaptée à la peinture avec un minimum de changement que devait permettre le passage à un portrait vivant. Un sourire est esquissé, les cheveux sont arrangés, les yeux sont ouverts et «ce que nous voyons comme un élément audacieux », l'excroissance sur la narine gauche, la maladie déformante du nez, a été modifiée. John Pope-Hennessy observe des différences

12. Lorne Campbell, Renaissance Portraits: European Portrait-Painting in the 14th, 15th and 16th Centuries, New Haven, Yale University Press, 1990, p. 190.

13. L'identité du vieillard n’a pas été découverte, mais le modèle éventuel avancé par plusieurs historiens serait Francesco Sacchetti. Voir l'article de Nathalie Volle, «La restauration du Vieillard et du jeune garçon de Ghirlandaio », La revue du Louvre, vol. 46, $\mathrm{n}^{\circ} 3$, juin 1996 , p. 51 . 
entre l'étude graphique et la représentation picturale: le dessin présente la tête de face alors que le portrait est de trois quarts, de même que la description des rides et du nez bosselé est plus insistante dans le dessin ${ }^{14}$. Selon l'auteur, tout semble indiquer que la maîtrise technique de Ghirlandaio est démontrée grâce à la figuration de la maladie jugée ressemblante et l'invention est ainsi reconnue pour la scénographie mise en place. Ces éléments confortent le statut du portrait à la Renaissance et la définition de l'art: la création est inséparable d'une exécution parfaite. La stratégie de l'apparaître et la figuration de la maladie redonnent vie à un corps mort. Nous retrouvons ici la «force divine» de la peinture selon Alberti, qui concourt à rendre présents les absents et à montrer les morts aux vivants. Il ajoute: «C'est donc que les visages des défunts prolongent d'une certaine manière leur vie par la peinture ${ }^{15} »$. La fortune critique du portrait de Ghirlandaio se comprend mieux alors, le portrait se présentant comme l'exemple même de la tâche artistique qui est celle de l'abolition de la mort.

\section{LA STRATÉGie de la NeUtralité AfFective}

Un autre procédé d'interprétation du tableau consiste à faire passer le motif pathologique pour un élément secondaire. Ainsi, le tableau de Ghirlandaio ne se limite plus à la représentation de l'acné hypertrophique du nez mais au fait de peindre la relation active entre deux personnes; cette interaction a été considérée comme sans précédent dans la peinture de l'époque. Selon Dominique Thiébaut, la fascination qu'exerce le portrait sur le public ne tient pas dans la représentation d'une "sévère maladie du nez décrite avec une précision presque "clinique"», mais plutôt dans la dimension affective du tableau : l'attitude confiante de l'enfant envers le vieillard, dont «la bonté et l'attendrissement font oublier la laideur ${ }^{16} »$. Tout cela manifeste la capacité étonnante de l'artiste à nous faire sentir la présence physique des personnages. Dans cette perspective, la dimension commémorative du portrait est affirmée par le fait qu'il immortalise les vertus de l'ancêtre, comme modèle de sagesse et de bonté.

14. John Pope-Hennessy, The Portrait in the Renaissance, Princeton, Princeton University Press, 1966, p. 56. Voir également John Shearman, pour son texte sur le portrait et sur l'idée de «création cérébrale », dans Only Connect. Art and the Spectator in the Italian Renaissance, Princeton, Princeton University Press, 1992, p. 108-110.

15. Leon Battista Alberti, De pictura, livre II, $\$ 25$, p. 131.

16. Dominique Thiébaut, «Un chef d'œuvre restauré: Le portrait d'un vieillard et d'un jeune garçon de Domenico Ghirlandaio (1449-1494)", La revue du Louvre, vol. 46, $\mathrm{n}^{\circ} 3$, juin 1996, p. $4^{2}$. 
Charles Rosenberg surenchérit à cet effet lorsqu'il affirme que la présence de l'enfant est là pour résoudre le problème qui se pose au peintre devant le modèle, à savoir comment parvenir à dégager la grandeur morale du défunt tout en respectant l'exigence de ressemblance, dans un contexte néoplatonicien où l'apparence physique indique la qualité de l'âme ${ }^{17}$. Selon Rosenberg, les traits du visage du modèle ont posé un problème au peintre, car le mandat du portrait est de rappeler l'apparence d'un personnage aux générations futures. Il y a donc deux demandes implicites faites à l'artiste: d'une part, l'image doit ressembler au modèle et d'autre part, elle doit désigner le caractère du modèle. Largumentation établie est la suivante: puisque la nature innée du personnage se révèle dans son apparence, seul un homme avec une belle âme peut commander le respect et obtenir l'amour d'un enfant, garant de la pureté. Ici, c'est bien l'innocence de l'enfant qui cautionne la présence de la pathologie. L'enjeu était alors de tenter de retrouver cette confiance accordée à l'image peinte. Le recours aux sentiments éprouvés par les personnages peints permet de nouveau d'esquiver la question de la pathologie en déplaçant le champ d'intérêt du tableau.

\section{LA SIDÉRATION DE L'ENFANT}

La pathologie du vieillard sert de preuve de la ressemblance aux yeux de certains historiens de l'art, car la ressemblance induit chez le spectateur la légitimité de la présence. Elle est totalement acceptée par les historiens de l'art, mais la condition de son acceptation est due à une modalité bien particulière : le modèle était mort au moment où a été réalisé le portrait, ce qui veut dire que la décision du peintre de figurer la déformation pathologique s'est produite sans le consentement du modèle. C'est la mort qui autorise la réalisation d'un tel portrait et qui conforte les historiens de l'art dans leur principe de convenance. Voilà donc la condition sine qua non du portrait en malade de Ghirlandaio: le peintre n'a plus à adopter la norme sociale étant donné que le modèle est mort et l'on peut supposer que la famille a alors commandité le portrait du vieillard. Pour les historiens de l'art, le portrait de Ghirlandaio rejoue le lieu commun voulant que le portrait soit la manifestation d'un désir de survie. Dans Du côté de chez Swann, Proust fait allusion au portrait de Ghirlandaio:

17. Charles Rosenberg, «Virtue, Piety and Affection. Some Portraits by Domenico Ghirlandaio », dans Augusto Gentili, Philippe Morel, Claudia Cieri Via (dirs.), Il ritratto e la memoria, materali 2, Rome, Bulzoni editore, 1993, p. 173-195. 
Swann avait toujours eu ce goût particulier d'aimer retrouver dans la peinture des maîtres non pas seulement les caractères généraux de la réalité qui nous entoure, mais ce qui semble au contraire le moins susceptible de généralité, les traits individuels des visages que nous connaissons: ainsi [...] sous les couleurs d'un Ghirlandaio, le nez de Monsieur de Palancy ${ }^{18}$.

Ce passage de Proust souligne que les artistes n'ont pas cherché à dissimuler les imperfections de la vie, ils ont inséré dans leur tableau « de tels visages qui donnent à la peinture un singulier certificat de réalité et de vie, une saveur moderne $^{19}$ ». Mais il pointe surtout le caractère particulier et distinctif de ce détail pictural, Swann regarde en face le détail incongru du portrait sans le contourner, de la même manière que la figure de l'enfant regarde et fixe de manière intense la curiosité épidermique qui se trouve devant ses yeux. Ce regard insistant que les 44 enfants abordent parfois devant la différence anatomique est un moment de sidération qui se manifeste comme le dédoublement du spectateur. Les yeux rivés de l'enfant sur ce qui provoque sa curiosité nous renvoient à notre propre sidération devant cette curiosité de la nature que la peinture donne à voir dans toute son évidence. Si le malaise des historiens de l'art se trouve dans l'interprétation de la déformation nasale, le véritable élément perturbateur de l'image réside, selon eux, dans le motif du paysage. Alors que Ghirlandaio est loué unanimement pour sa capacité à imposer l'évidence d'une présence au moyen du naturalisme, par sa connaissance du triptyque Portinari, les historiens de l'art n'expliquent pas le traitement du paysage, jugé archaïque. Ce motif court-circuite le discours des historiens de l'art justement parce qu'il s'agit d'une figure surdéterminée; la colline jonchée d'arbres aux feuillages sphériques reprend les aspérités du nez déformé. Par ce motif, le dispositif visuel réaffirme son parti pris d'exposer la pathologie comme une opération défensive de la peinture pour mieux indiquer qu'il faut voir la déformation, la voir deux fois afin de ne pas l'esquiver.

Les diverses appréciations du portrait de Ghirlandaio rejouent la confusion qui s'est installée entre la qualité d'une image picturale et l'aspect physique de la personne représentée, et qui pointe directement vers l'enjeu philosophique du portrait en malade. C'est l'idée que la supériorité de l'image est due à sa belle description de choses qui, dans la nature, sont laides ou déplaisantes. Rappelons que Kant souligne que la maladie peut être décrite d'une belle manière, elle peut même être représentée en peinture, du moment qu'elle ne suscite pas un affect négatif comme le dégoût: «Seul un certain genre de laideur ne peut être représenté d'après nature sans ruiner toute satisfaction esthétique, donc la

18. Marcel Proust, Du côté de chez Swann, Paris, Éditions Gallimard, 1988 [1913], p. 219. 19. Marcel Proust, Du côté de chez Swann, p. 220. 
beauté artistique : la laideur qui provoque le dégoût ${ }^{2 \circ}$ ». L’important est donc que la satisfaction esthétique prône sur l'affect négatif, c'est-à-dire qu'elle ne provoque pas une supériorité de l'affect comme la répulsion. Les critères kantiens secondent les analyses du portrait de Ghirlandaio, légitimant de manière sous-jacente l'interdit théorique de la figuration du disgracieux et du laid. À la suite de cette constatation, nous dirons alors, peut-être de manière un peu provocatrice, que vis-à-vis des discours de l'histoire de l'art, la figuration de la maladie porte un masque, comme si un réflexe spontané de détournement de l'image se produisait à partir d'un discours de la vérité de l'apparence. L'argumentation de la prouesse du peintre se manifeste comme le masque déposé sur la figuration de la pathologie. Tout se passe comme si le discours critique tendait à contourner, à oblitérer les figures pathologiques de la peinture en affirmant que seule compte la qualité du travail artistique. Ainsi, la représentation de la maladie n’apparaît pas comme une source d'inquiétude ou de malaise et elle n'est pas non plus considérée comme une forme déplaisante. En fait, le mot de passe est «naturalisme» et la stratégie consiste à vanter la prouesse picturale pour mieux refuser d'abandonner son regard à la figuration du pathologique lorsque ce dernier est présenté dans un aspect descriptif. Ghirlandaio n'a donc pas commis de crime de lèse-majesté en portraiturant une maladie déformante dans un portrait, car la mort du modèle lui donnait cette licence et les historiens de l'art accueillent cette liberté picturale au nom de la ressemblance.

\section{LA PESTE DE LA PEINTURE}

Dans le discours de l'histoire de l'art, un peintre à lui seul témoigne de la maladie de la peinture. Rappelons ici les propos de Félibien : «M. Poussin ne pouvait rien souffrir du Caravage et disait qu'il était venu au monde pour détruire la peinture $»^{21}$. Autant dire que Caravage est le fléau de la peinture, la peste du grand art. Voilà un peintre qui s'est autorisé à tout peindre et qui a même été jusqu'à se peindre malade, selon l'interprétation qui a été faite d'un de ses tableaux. Le Bacchus malade du Caravage doit son nom à l'ambiguité produite par la carnation du personnage représenté. Le titre du Bacchus malade est une proposition de Roberto Longhi formulée en 1927 lorsqu'il interprète le tableau comme un

20. Emmanuel Kant, Critique de la faculté de juger, suivi de Idée d'une histoire universelle au point de vue cosmopolitique et de La réponse à la question: qu'est-ce que les Lumières?, trad. Alexandre J. L. Delamarre, Jean-René Ladmiral, Marc B. de Launay, JeanMarie Vaysse, Luc Ferry et Heinz Wismann, Paris, Éditions Gallimard, 1985, p. $267, \int 48$.

21. Cette citation de Félibien, dans Entretien sur les vies et les ouvrages des plus excellents peintres, entretien VI, 1685 , est longuement commentée par Louis Marin dans Détruire la peinture, Paris, Galilée, 1977, p, 14, 41, 119, 128 et 130. 
autoportrait du Caravage, en partant du témoignage de Giulio Mancini. Dans son Trattato della pittura, Giulio Mancini avait rappelé une maladie du peintre et son séjour à l'Hôpital de la Consolation à Rome. Il ajoute que durant sa convalescence, Caravage aurait peint un grand nombre de tableaux pour le prieur de cet hôpital ${ }^{22}$. Roberto Longhi voit dans le Bacchus malade, aujourd'hui au palais Farnèse, «les traits du peintre adolescent et encore en mauvaise santé23». La discipline de l'histoire de l'art a adopté le titre établi par Roberto Longhi malgré le fait que cette interprétation n'ait pu être authentifiée ${ }^{24}$. Certains auteurs ont évoqué la syphilis comme preuve des comportements de l'artiste jugés luxurieux renforçant ainsi le jugement négatif porté sur son œuvre. En outre, des pathologies autres que la syphilis ont été reconnues dans le Bacchus malade du Caravage : Herwart Röttgen observe, dans ce portrait, les symptômes de la mélancolie comme contre-figure de la vie, au même titre qu'une vanitas ${ }^{25}$.

\section{SUSTERMANS OU LES EFFETS ÉPIDERMIQUES DU PICTURAL}

La stratégie dissimulatrice de la peinture, défendue par l'historiographie, n'a pas été la préoccupation de tous les artistes. Des peintres ont traité le portrait en malade comme une exigence supplémentaire de la peinture, en dépit de laquelle ils ont poursuivi la seule expérience qui leur importait, à savoir le travail de la matière colorée. Le peintre met alors en évidence l'excès qu'a produit un état pathologique, il témoigne d'une angoisse face au corps en défaut qu'il n'a pas tenté de masquer. Arrêtons notre regard sur le portrait réalisé par Giusto Sustermans

22. Le passage de Giulio Mancini est cité par Roberto Longhi, Studi caravaggeschi. 1935-1969, Milano, Sansoni, 2000, tome 2, p. 33.

23. Roberto Longhi souligne «il corpo di questo giovinetto è di una tonalità eccessivamente gialla come di chi sia appena uscito da un periodo di febbri palustri.», Roberto Longhi, Saggi e ricerche: 1925-1928, Firenze-Sansoni, 1967, tome 2, p. 304. Roberto Longhi, Le Caravage, trad. Gérard-Julien Salvi, Paris, Éditions du Regard, 2004, p. 27.

24. Marco Gallo retrace les diverses interprétations de cette œuvre dans Sergio Rossi (dir.), Scienza e miracoli nel l'arte del' 6oo. Alle origini della medicina moderna, catalogue d'exposition, Milan, Electra, 1998, p. 327. Voir aussi Marco Gallo, «Materiali per Caravaggio. Bibliografia di Michelangelo Merisi 1980-1996», dans Stefania Macioce (dir.), Michelangelo Merisi da Caravaggio. La vita e le opere atravverso i documenti, Atti del convegno internazionale di studi, Roma, Logart Press, 1997. Voir également Flavio Caroli (dir.), L'animo e il volto. Ritratto e fisiognomica da Leonardo a Bacon, Milan, Palais Reale, 30 octobre-14 mars 1999, Milan, Electra, 1998, p. 180.

25. Voir Herwart Röttgen, Il Caravaggio. Ricerche e interpretazioni, Rome, Bulzoni Editore, 1974, p. 183. 


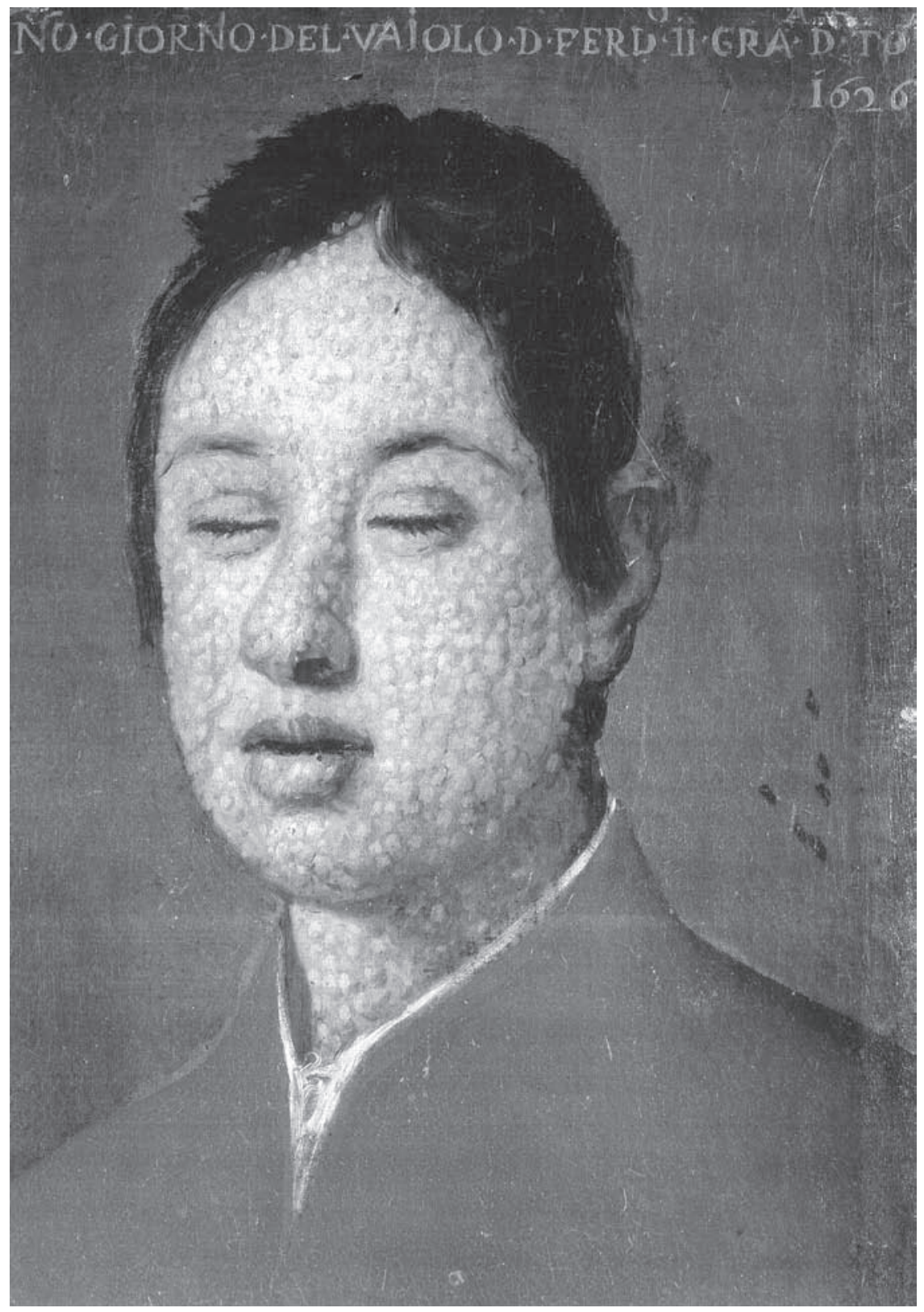

Fig. 5. Giusto Sustermans, Portrait du grand-duc Ferdinand II de Medicis, 1626, huile sur toile, 43 x $33 \mathrm{~cm}$, Florence, Palazzo Pitti, Galleria Palatina. (C) Avec l'aimable autorisation du Ministero dei Beni e le Attività Culturali. 
lors de la variole du grand-duc de Toscane, Ferdinand II. Le peintre a produit trois portraits réalisés à quelques jours d'intervalles, portraits qui témoignent de l'évolution de la variole. Le portrait du palais Pitti est le dernier réalisé, il porte l'inscription suivante sur le bord supérieur du tableau: "Il granduca, durante la grave malattia, fu ritratto dal pittore di corte il $3^{\circ}, 6^{\circ}$ e il $9^{\circ}$ giorno ». Le premier semble perdu, le second représente le grand-duc dans les premiers jours de la maladie avec une apparition de pustules, alors que le troisième portrait illustre l'acmé de la maladie, lorsque les yeux gonflés par les pustules ne peuvent plus s'ouvrir (fig. 5). La variole du grand-duc est un prétexte à la picturalité : les pustules sont traités par petites touches de couleurs flottantes. Le contraste violent des cheveux et du vêtement permet de travailler le visage en teintes fondues sans rupture apparente, des coups de brosse réguliers laissent affleurer l'épaisseur de la pâte. L'image joue sur le contraste entre le lissé du vêtement de couleur rouge et le visage traité en larges touches de pinceau appliquées l'une à côté de l'autre puis complétées par des retouches qui ne se cachent pas. L'effet de proximité extrême avec le spectateur est accentué par le très gros plan du visage que nous suggère Sustermans. Les deux portraits de Ferdinand II malade trouvent une légitimité aux yeux des historiens de l'art lorsqu'ils sont compris au regard de leur contexte sociohistorique, c'est-à-dire la cour du grand-duc. Interprétés comme une exceptionnelle mémoire scientifique, les portraits s'expliquent par le fait que le modèle était un personnage de grande liberté, ami des artistes, amoureux des sciences, et qu'il avait lui-même un laboratoire dans lequel il procédait à certaines expériences. Louverture d'esprit du grand-duc de Toscane, Ferdinand II, et le contexte de la cour des Médicis à cette époque, particulièrement dans le domaine des arts, permettent de proposer qu'il y ait dans l'œuvre de Giusto Sustermans une volonté de démonstration clinique ${ }^{26}$. Le souci de représentation dans ce cas singulier du portrait vise à témoigner d'un fait pathologique dans toute son évidence. Il s'agit ici de faire rentrer cette image dans un cadre théorique selon lequel elle répond à une finalité.

\section{LE PORTRAIT DE LA VARIOLE}

Le double portrait réalisé par Sustermans déborde le modèle représentatif dominant, il en marque toutes les limites et oblige à s'interroger sur la nature et les objectifs de la figuration humaine. Dans ce cas très particulier de représentation de la variole, l'évolution de la maladie figurée par le principe de la succession

26. Voir Marco Chiarini, Claudio Pizzorusso (dirs.), Sustermans: sessant'anni alla corte dei Medici, catalogue d'exposition, Florence, Palais Pitti, Centro Di, 1983. 
des portraits renvoie à notre propre condition humaine, en permanente transformation. Il va donc à l'encontre du principe même du portrait qui est de présenter le caractère durable d'un personnage, alors que l'artiste choisit de représenter l'impression d'un instant. Le caractère par définition invariable de la figuration humaine est mis à mal par les portraits de Sustermans. Les deux états figurés ne sont que transitoires et l'état du visage ne nous parle que de ses variations, de sa métamorphose par le processus de la maladie: c'est une mémoire ouverte ${ }^{27}$.

En poussant l'argumentation plus loin, nous dirions que le double portrait du grand-duc Ferdinand II n'est pas un portrait en tant que tel, ce n'est pas le portrait de Ferdinand II de Médicis qui nous est donné à voir mais plutôt le portrait de la variole. C'est la représentation d'un visage observé comme mémoire de la maladie dans laquelle la figure s'éclipse. La maladie apparaît alors comme une expérience qui met en cause le visage humain et ses facultés expressives, étant donné que le traitement pictural de la figure tend à le noyer dans l'informe. Sustermans a su donner une légitimité mimétique de la variole du grand-duc. Ce qui fait la force de la représentation, c'est sa puissance d'effets et sa violence d'affects. La visibilité du visage étant entamée, elle se désintégre derrière la maladie. C’est donc une présence humaine qui s'affiche mais sous l'aspect d'une face travaillée par le processus de la variole. L'effacement du visage est réalisé pour faire surgir la maladie car il s'agit bien de peindre le processus même de la maladie et donc le caractère mouvant et énergétique de toute pathologie.

Les deux portraits de Sustermans ont une singulière façon de remettre en question le primat de la représentation, car le portrait ne dévoile pas une physionomie et la ressemblance mimétique n'est pas la propriété essentielle du portrait en malade. Cette incomplétude du visage due à la maladie tient aussi à la structure picturale qui est traitée par touches disposées sur la toile. L'accent mis sur la facture et les composantes matérielles de l'image était susceptible de contrarier la synthèse «imageante» et la lisibilité des figures. Ce type de tactilité et cette présence intempestive de la variole à la surface du tableau nous permettent de dire que c'est la peinture elle-même, dans son statut de matière, qui nous est donnée à voir. L’image de la picturalité se manifeste alors comme le caractère par excellence de la peinture, telle une peau, simultanément pellicule de surface et réseau d'échanges avec la profondeur.

27. Récemment, les médias nous ont montré un processus similaire de la maladie à travers les portraits de Victor Iouchtchenko, le chef de l'opposition ukrainienne, dont le visage a été grêlé par une acné chlorique dû à un empoisonnement à la dioxine. Voir l'article de Dominique Dhombres, «Le pays dont le héros a le visage ravagé par l'acné», Le Monde, 25 novembre 2004 . 
Dans le cas du portrait du grand-duc, nous pouvons dire que les données fondatrices de l'image humaine sont falsifiées au profit d'une représentation du devenir du corps qui provoque la sidération. Le portrait de Sustermans est l'antithèse des portraits de Bronzino, de la figure idéalisée sans aspérité, il déclenche un sentiment de répulsion envers le corps pustuleux, rugueux d'aspérités. Le visage du grand-duc n'est pas proposé au regard, il est imposé à la vue. Il stoppe la mobilité du regard, d'habitude prospectif dans la peinture. L'image de Sustermans parie sur la surprise car l'œil médusé est incapable de s'arracher à ce qui le sidère. Il s'agit de faire éprouver un affect de la limite, un affect du débordement. Sustermans a peint la variole avec le trouble qu'elle entraîne.

Cette vérité des yeux de voyant du peintre, en face de quelqu'un qui ne voit plus, renvoie à la situation d'une autre image; le portrait de Luigi Groto, un poète aveugle, peint par le Tintoret. Le modèle aveugle en train de se faire peindre, place l'artiste et, bien sûr, le spectateur, dans une position de voyeurs. Une intimité nous est dévoilée que nous ne sommes pas certains de pouvoir soutenir. C'est peut-être là toute l'histoire du rapport que nous avons avec l'image de la pathologie. Le portrait réalisé par Sustermans nous montre que la maladie a obligé le modèle à clore les yeux alors que la peinture a aveuglé le spectateur au moyen de la touche picturale. Dans son effet de tactilité, la peinture nous masque le portrait du grand-duc pour mieux nous exposer la pathologie.

Dans l'histoire de l'art, la pathologie a trouvé plus souvent un espace par le recours à la métaphore que dans l'étude de la représentation de la maladie. Au XIX ${ }^{\mathrm{e}}$ siècle, la critique d'art a usé très largement de métaphores pathologiques pour disqualifier le travail des Impressionnistes ${ }^{28}$. Les métaphores de la critique d'art font état d'un médium en proie à une maladie épidermique, à des éruptions cutanées de la peinture, cela en vue de révéler la matérialité picturale des images qui s'évalue autour des termes d'eczéma, de croûtes jusqu'à constituer un lieu commun de l'indignation et de l'injure ${ }^{29}$. Lorsqu'il s'agit de l'interprétation du

28. Voir sur ce point Nicole Dubreuil-Blondin, «Les métaphores de la critique d'art: le "sale" et le "malade" à l'époque de l'impressionnisme », dans Jean-Paul Bouillon (dir.), La critique d'art en France. 1850-1900. Actes du colloque de Clermont-Ferrand, 25, 26 et 27 mai 1987, Saint-Étienne, CIEREC, Université de Saint-Étienne, 1989, p. 96-105. Également, Michel Ribon, Archipel de la laideur. Essai sur l'art et la laideur, Paris, Kimé, 1995.

29. Dans son essai La peinture incarnée, Georges Didi-Huberman a montré l'impressionnante circulation de métaphores entre le corps et le tableau. Il développe l'idée de l'image comme figure par excellence de la peinture, puisqu'elle se donne simultanément comme une pellicule, une surface mais aussi comme un réseau d'échanges avec la profondeur. Georges Didi-Huberman, La peinture incarnée, Paris, Éditions de Minuit, coll. «Critique», 1985, p. 35-44. 
tableau, le recours à la pathologie sert parfois à exprimer une dissonance de la peinture. Le Bacchus malade du Caravage doit son nom à la difficulté d'interpréter la couleur de la carnation du personnage représenté.

Unanimes sur la question du portrait, les théoriciens de l'art de la Renaissance conviennent que le peintre doit refigurer son modèle sans jamais le défigurer ${ }^{30}$. La condamnation sans appel de la déformation du visage trouve son explication dans la conviction culturelle qui fait du visage le lieu de l'esprit et de l'identité, mais elle corrobore également la conception privilégiée de la suprématie du visage et de son statut particulier, essentiel dans l'évaluation occidentale de la beauté et de la décrépitude physique. Face aux portraits en malades, les historiens de l'art se sont surtout interrogés sur la nature du projet artistique. La curiosité initiale que produit le portrait d'un malade exigeait de comprendre comment de telles œuvres avaient pu voir le jour. Comment le portraitiste, aux prises avec les règles d'un système représentatif socialement codifié, avait pu produire un déni des conventions. Cherchant avant tout à justifier les portraits pathologiques, l'historien de l'art ne s'est pas attardé sur la sidération du portrait, sur cette irruption d'une violence qui vient bousculer la figuration dans son procès de symbolisation. Pourtant, dans la représentation d'un visage pathologique, ce qui est représenté, ce n'est pas une forme dans laquelle nous devons rechercher une vraisemblance, c'est une puissance d'effet du tableau à recevoir et à soutenir.

30. Sur la question de la littérature artistique et sur les préceptes donnés aux artistes quant à la représentation du portrait, nous renvoyons le lecteur à notre thèse Les images des maux. Étude de la représentation de la pathologie dans l'art de la Renaissance, École des Hautes Études en Sciences Sociales, sous la direction de Daniel Arasse, 2005. 